Conclusion: Because US DM fellowships are non-ACGME accredited, there is a lack of conformity in their educational models. This study provides applicants with the differentiating data needed to make educated decisions on which is the best fit for them.

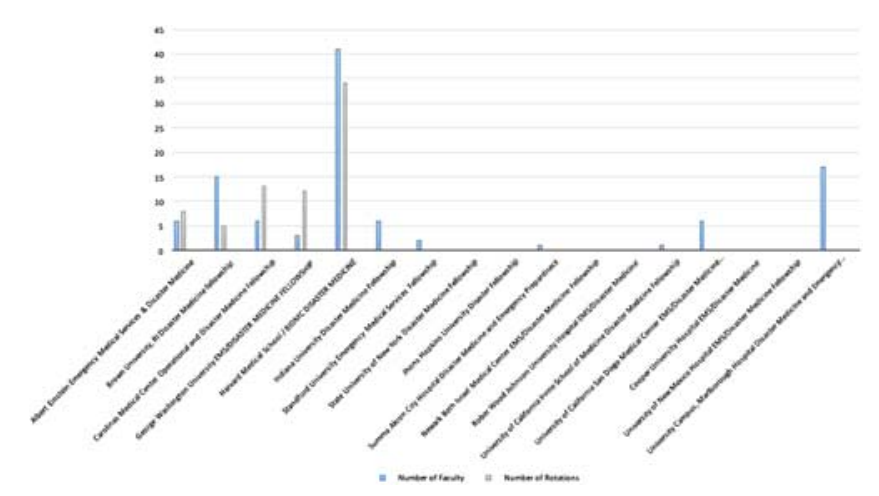

Prehosp Disaster Med 2017;32(Suppl. 1):s26-s27

doi:10.1017/S1049023X17000899

\section{Disaster Severity Index: Proposal of a New Tool in}

Disaster Metrics

Ying Ying Yerw, Pedro Arcos Gonzalez, Rafael Castro Delgrado

Unit For Research In Emergency And Disaster. Department Of Medicine, Universidad De Oviedo, Oviedo/Spain

Study/Objective: The Richter Scale measures the magnitude of a seismic occurrence, but does not feasibly quantify the magnitude of the "Disaster" at the point of impact in real humanitarian needs based on United Nations International Strategy for Disaster Reduction (UNISDR) 2009 Disaster Terminology. A Disaster Severity Index similar to Richter Log Algorithm has been formulated; this will quantify needs, holistically, and objectively, in the hands of any stakeholders and even across timelines.

Background: An agreed terminology in quantifying "Disaster" matters, and inconsistency in measuring it by stakeholders, posed a challenge globally in formulating legislation and policies responding to it.

Methods: A mathematical calculation which uses the median score percentage of $100 \%$ as a baseline, indicating the ability to cope within the local capacity. Seventeen indicators were selected based on the UNISDR 2009 disaster definition of vulnerability and exposure and holistic approach as a precondition. The severity of the disaster is defined as the level of unmet needs. 30 Natural disasters were tested retrospectively and non-parametric tests were used to test the correlation of the Disaster Severity Index scored against the Indicators.

Results: The findings showed that 20 out of 30 Natural Disasters tested fulfil the inability to cope within local capacity in Disaster Terminology. Non-parametric tests showed that there is a correlation between the 30 Disaster Severity Index Scored and the Indicators.

Conclusion: By computing a median fit percentage score of $100 \%$ as the ability to cope, and the correlation of the
17 indicators in this Disaster Severity Index Scale, 20 natural disasters fit into the Disaster definition. This Disaster Severity Index will enable humanitarian stakeholders to measure and compare the severity of the disaster objectively and enable future response to be based on needs.

Prehosp Disaster Med 2017;32(Suppl. 1):s27

doi:10.1017/S1049023X17000905

Microchips, from a Disaster Perspective

Reem B. Alfalasi, Michael Molloy, Amalia Voskanyan, Ritu Sarin, Gregory Ciottone

The Bidmc Fellowship In Disaster Medicine, Department of Emergency Medicine, Beth Israel Deaconess Medical Center, brookline/AL/United States of America

Study/Objective: To review the documented uses of microchips in the medical field, and explore their possible utilisation in the disaster medicine environment.

Background: Microchips have a number of non-medical uses in varied fields including banking, retail and the veterinary sectors. In some countries it is mandatory to chip domestic pets to enable identification if they stray from home. Disaster preparedness organisations in the US advocate chipping animals to facilitate post-disaster reunification. To date there is limited data on use of microchips in the field of disaster medicine or the ethical implications of their use.

Methods: The authors performed a review of literature indexed in PubMed and the Cochrane Library with no limits on year of publication or language, including both human and animal results. Exploded search terms included "microchip"” Biochip"” "RFID"” "Disaster RFID" tracking and/or identification.

Results: Search strategy yielded 686 citations, with 40 records used in this review, 9 from the veterinary field and 31 from the medical field. These papers suggested multiple existing uses of the microchipping technology, including identification, the retrieval of medical information in the event of an emergency and the use of GPS-enabled chips in locating missing individuals, a few of which can be used in the setting of a disaster.

Conclusion: Based on the results of the study, several different uses of this technology were identified . Microchips have proven to be beneficial in tracking and identification, in both the medical and the veterinary medicine field. This paper aims to explore this topic further by looking at the current uses of microchips, and by suggesting additional uses of this technology in the disaster setting, such as triaging and patient identification.

Prehosp Disaster Med 2017;32(Suppl. 1):s27

doi:10.1017/S1049023X17000917

It's a crush... It's a collapse... It's... Wait, that's No Stampede!

Sravani Alluri, Amalia Voskanyan, Ritu R. Sarin, Michael S. Molloy, Gregory R. Ciottone

The Bidmc Fellowship In Disaster Medicine, Department of Emergency Medicine, Beth Israel Deaconess Medical Center, Boston/MA/United States of America 